\title{
Metodologías para reforzar la literacidad y el respeto por los libros
}

\author{
Methodologies to Strengthen Literacy and Respect for Books
}

\section{Natalia Tello Burgos}

tellonatalia@gmail.com

Ana Isabel Calero Castillo

anacalero@ugr.es

Ana María López Montes

alopezmontes@ugr.es

Universidad de Granada (España)
Recibido 14/09/2020 Revisado 10/10/2020

Aceptado 11/10/2020 Publicado 31/10/2020

\section{Resumen:}

A partir del año 2017, desde el Departamento de Pintura de la Universidad de Granada dentro del Grado en Conservación y Restauración de Bienes Culturales, estamos realizando actividades dirigidas a fomentar la literacidad en el alumnado y recuperar la apreciación por los libros a diferentes edades. Con la incorporación de las nuevas tecnologías y el acceso a contenidos de todo tipo a través de las pantallas, asistimos al paulatino arrinconamiento de la lectura y el empleo de libros, restándoles valor y atención a sus aspectos formales, decorativos y textuales. Es por ello que consideramos fundamental llevar a cabo este tipo de iniciativas que trasladan a las aulas actividades prácticas para impulsar el conocimiento y reconocimiento de los libros a la par que desarrollan habilidades motrices, cognitivas y su creatividad.

Debido a las inusuales circunstancias de este año 2020 y con motivo de la celebración del día del libro el 23 de abril, se han propuesto actividades para poder realizar en casa mediante un archivo digital descargable con contenido visual y audiovisual. Para la divulgación y la exposición de los trabajos realizados se ha utilizado un hashtag y las redes sociales.

La realización de este tipo de actividades está aportando resultados muy positivos y con una alta implicación de los colectivos participantes. Además, consideramos que la implementación de este tipo de actividades de manera regulada en la enseñanza aporta numerosas ventajas como la sensibilización y conocimiento del patrimonio documental, la importancia de su conservación y su defensa.

\section{Sugerencias para citar este artículo,}

Tello Burgos, Natalia, Calero Castillo, Ana, Ana María López Montes, (2020). Creando historias: metodologías para reforzar la literacidad y el respeto por los libros. Tercio Creciente (Monográfico extraordinario II), págs. 89-102, https://dx.doi.org/10.17561/rtc.extra2.5754

TELLO BURGOS, NATALIA, CALERO CASTILLO, ANA, LÓPEZ MONTES, ANA MARÍA. Creando historias: metodologías para reforzar la literacidad y el respeto por los libros. Tercio Creciente (Monográfico extraordinario II) octubre 2020 pp. 89-102, https://dx.doi.org/10.17561/rtc.extra2.5754 


\section{Abstract:}

From 2017, the Department of Painting of the University of Granada, within the Degree in Conservation and Restoration of Cultural Heritage, carries out activities aimed at promoting literacy among students and recovering the appreciation of books at different ages. With the incorporation of new technologies and the access to information through the screens, we assist in the gradual abandonment of reading and the use of books, reducing their value and attention to their formal, decorative and textual aspects. That is why we consider it essential to carry out this type of initiative that brings practical activities into the classroom to promote knowledge and recognition of books while developing motor and cognitive skills and creativity.

Due to the unusual circumstances of this year 2020 and for the celebration of Book Day on 23rd April, activities have been proposed to be carried out at home by means of a downloadable digital file with visual and audiovisual content. A hashtag and social networks have been used to disseminate and exhibit the work done.

The realization of this type of activities is bringing very positive results and with a high implication of the groups involved. Furthermore, we consider that the implementation of this type of activities in a regulated way in education brings many advantages such as the awareness and knowledge of the documentary heritage, the importance of its conservation and its defence.

Palabras Clave: Patrimonio, libros, literacidad, educación, conservación. Key words: Heritage, books, literacy, education, conservation.

Sugerencias para citar este artículo,

Tello Burgos, Natalia, Calero Castillo, Ana, Ana María López Montes, (2020). Creando historias: metodologías para reforzar la literacidad y el respeto por los libros. Tercio Creciente (Monográfico extraordinario II), págs. 89-102, https://dx.doi.org/10.17561/rtc.extra2.5754

TELLO BURGOS, NATALIA, CALERO CASTILLO, ANA, LÓPEZ MONTES, ANA MARÍA. Creando historias: metodologías para reforzar la literacidad y el respeto por los libros. Tercio Creciente (Monográfico extraordinario II) octubre 2020 pp. 89-102, https://dx.doi.org/10.17561/rtc.extra2.5754 


\section{Introducción}

Desde la facultad de Bellas Artes y en concreto desde el Grado en Restauración y Conservación de Bienes Culturales de la Universidad de Granada se trabaja e investiga sobre los libros como soporte material, atendiendo a su grado de conservación y asegurando su prevalencia en el tiempo. Mediante una serie de asignaturas estrechamente relacionadas con los libros, sus características, historia y estado de conservación, nuestro trabajo como docentes e investigadores nos ha hecho reflexionar sobre cuestiones relacionadas con la lectura y el uso de los libros desde la etapa preescolar y hasta niveles universitarios.

Vivimos en la Era Digital donde nuestro contexto social está muy interrelacionado con las nuevas tecnologías. Debido al uso progresivo de la tecnología (ordenadores, móviles, tablets) estamos asistiendo al abandono paulatino de la lectura en general (Millán, 2017), pero sobre todo al reemplazo de los libros físicos, es decir, la sustitución del soporte papel por dispositivos electrónicos donde jugar o sentirnos "conectados" (Gil, 2011). La lectura no sólo cumple una función de entretenimiento y conocimiento, sino que es un proceso activo que contribuye al desarrollo de la comprensión y la interpretación de ideas escritas (Argüelles, 2018). De esta manera el lector pone en funcionamiento sus conocimientos previos, así como sus destrezas y habilidades cognitivas, metacognitivas y comunicativas en general. Por esta razón, consideramos que nuestro deber como docentes e investigadores implicados en el mundo del libro, es potenciar el uso de los libros apoyando el desarrollo de la competencia lectora de los individuos. Así, desde nuestro equipo de trabajo de investigadores y docentes de educación superior trabajamos para transferir el conocimiento que se genera en el ámbito universitario y compartirlo con la sociedad en su conjunto a través de los más pequeños (López, 2014).

Por todo esto, desde el año 2017, venimos impulsando la realización de talleres con el objetivo de promover diversas actividades dirigidas a fomentar la literacidad en el alumnado de infantil, primaria y secundaria y así, recuperar la apreciación por los libros a diferentes edades con la intención de impulsar el conocimiento y el reconocimiento de los mismos a la vez que desarrollar las habilidades motrices, cognitivas y la creatividad de los estudiantes.

Actividades de estas características las hay muy variadas, aunque generalmente se han orientado a personas jóvenes o de mediana edad que se movían por un interés personal. Además, han sido consideradas como "manualidades" para el entretenimiento o la "creación artesanal" de artículos personales.

En esta ocasión el reto es despertar el interés por los libros a todo el colectivo (haya mostrado interés previo o no), y demostrar la necesidad de un estudio profundo sobre su creación para poder valorarlo en su justa medida y poder realizar creaciones sólidas y funcionales y no meramente estéticas. 
Contenido y continente deben formar un conjunto estable, armónico y coherente. Valores que tras los talleres serán capaces de identificar en los libros que suelen consultar (académicos, novelas, cuentos...). Estas tres características deberán de ir incluyéndose de manera tímida en los talleres propuestos, donde la temática de la pieza, el diseño y el desarrollo de la obra deben de estar planificados desde el inicio del trabajo.

\section{Talleres presenciales}

Todas las actividades que realizamos en el ámbito docente se realizan en talleres, hasta ahora, de carácter presencial de una duración máxima de 1 hora, en los que mediante diferentes modalidades de actividades damos a conocer nuestro trabajo y el mundo del libro.

Estos talleres están impulsados por el profesorado del Grado en Restauración y Conservación de Bienes Culturales, que involucra tanto a estudiantes como a egresados para su realización. En todos los casos las actividades que realizamos son gratuitas para el alumnado que asiste, y fundamentalmente se han desarrollado en centros educativos de la provincia de Granada.

Las acciones que realizamos en ellos suelen dividirse por edades, adaptando el contenido, dependiendo de los asistentes, ya que todas ellas tienen en cuenta las características del alumnado al que van dirigidas, así como las posibles barreras o limitaciones que pudieran surgir durante su desarrollo.

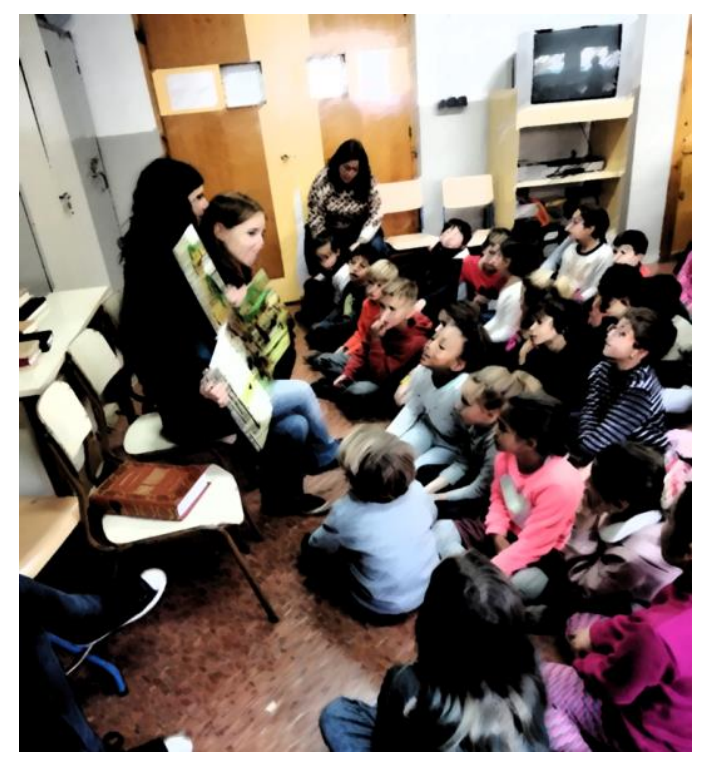

Ilustración 1: Imagen de la disposición del alumnado en uno de los talleres ofrecidos al CEP El Carmelo de Granada/ Fotografía: Ana Isabel Calero Castillo. 
Normalmente la estructura de la clase se realiza colocando a los niños y niñas formando un círculo a nuestro alrededor, sentados en el suelo para que puedan vernos mejor (Ilustración 1). En todos los casos, el taller se inicia con un acercamiento hacia el libro, exponiendo su evolución hasta la actualidad, mostrándoles las distintas tipologías, las partes principales que los conforman $\mathrm{y}$, con ejemplos prácticos, diferentes trucos para su cuidado y conservación en el tiempo con el objetivo de evitar su deterioro; algo que para nuestro equipo es fundamental debido a la investigación y labor docente en la que trabajamos (Tacón, 2009).

Después de esta primera parte de carácter teórico-participativo, se realiza una de las tipologías de actividad práctica, adecuada a su nivel educativo, así como al nivel de aprendizaje de cada alumno que participa.

\subsection{El libro acordeón}

Esta actividad va dirigida al alumnado de infantil entre 3 y 5 años de edad, aunque también, con algunas variaciones, añadiendo cierta dificultad y complejidad se puede realizar por alumnado de entre 5 y 7 años.

El material requerido para llevar a cabo esta actividad es principalmente cartulinas, rotuladores y pintura de dedos. Las cartulinas se utilizan, en el caso de los más pequeños previamente recortadas, en formato horizontal para que los niños y niñas las decoren con la pintura simulando hierba y flores. Durante el proceso tanto sus profesores como los encargados del desarrollo de la actividad, colaboramos con ellos para ayudarles a doblar el formato y terminar de decorarlo.

En el caso de los más mayores (alumnos de 5 a 7 años) se les incorpora algo más de complejidad ya que pueden emplear los materiales con más facilidad y de manera más precisa, utilizando rotuladores en vez de pintura de dedos y añadiendo más detalles a las escenas. Además, se les invita a que añadan tapas a su libro acordeón, así como un título para su cuento.

Una vez finalizada la actividad, tanto a los participantes más pequeños como a los más mayores, se les da la oportunidad de contar la historia que contiene su libro al resto de sus compañeros y compañeras (Ilustración 2). De esta manera el alumnado tiene la posibilidad de compartir con los demás las creaciones realizadas en el taller, fomentando además el desarrollo de sus habilidades sociales al exponer su trabajo para todo el grupo. 
ISSN: 2340-9096

https://dx.doi.org/10.17561/rtc.extra2.5754

Una de las variantes que en ocasiones se ha introducido en esta actividad y que ha resultado muy positiva, ha sido la de incorporar a la tarea a un grupo mayor del mismo colegio (de $1^{\circ}$ a $4^{\circ}$ ESO). El objetivo en este caso ha sido el de implicar a los alumnos de esta etapa con los más pequeños, de manera que asistiendo a la primera parte de la actividad teórica en círculo y atendiendo a las directrices de las personas que presentan la actividad, son ellos los que se encargan de ayudar a los más pequeños en la realización de su libro acordeón. Esta forma de trabajar hace responsables de manera indirecta al grupo más mayor (ESO) de los más pequeños (infantil) resultando con ello, el intercambio de conocimiento y un excelente comportamiento por parte de ambos grupos (Ilustración 2).

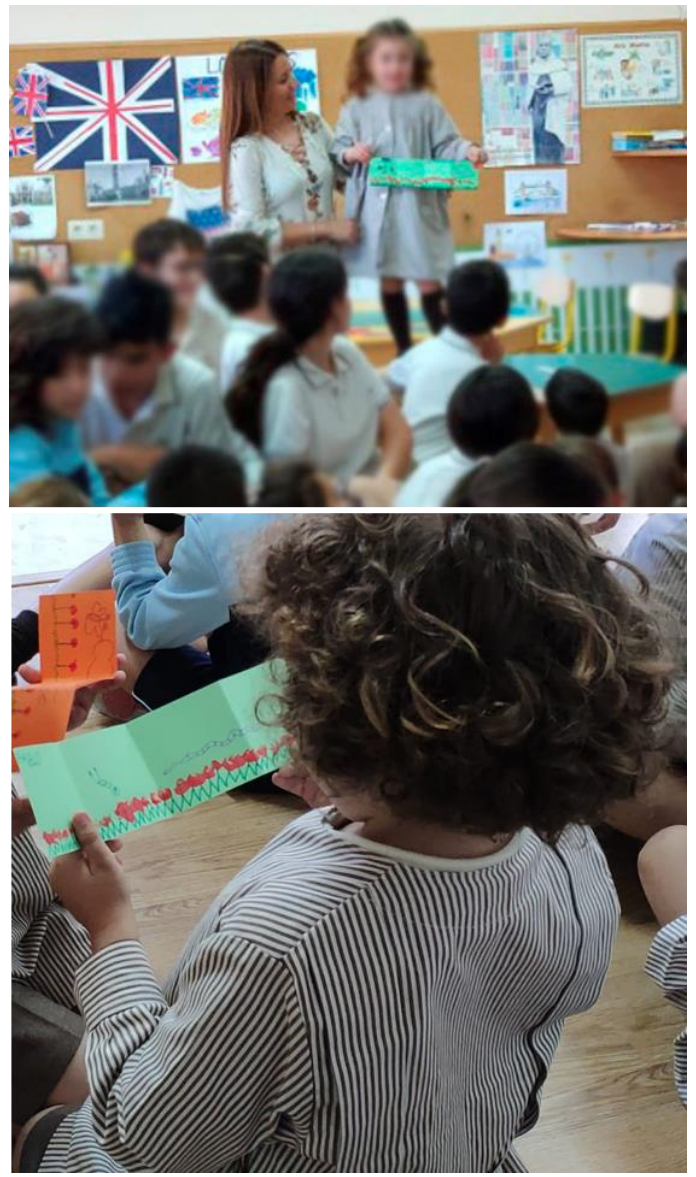

Ilustración 2: Alumnos de primaria y secundaria participando en la actividad del Libro acordeón. CEP El Carmelo de Granada/ Fotografía: Ana María López Montes. 


\subsection{El mapa del tesoro}

Esta actividad se denomina el mapa del tesoro y va dirigida al alumnado con una edad comprendida entre los 8 y los 12 años. Los materiales para realizar este tipo de libro pueden ser muy variados dependiendo de lo que prefiera cada estudiante en los que el mismo puede desarrollar su creatividad a partir de un modelo concreto propuesto.

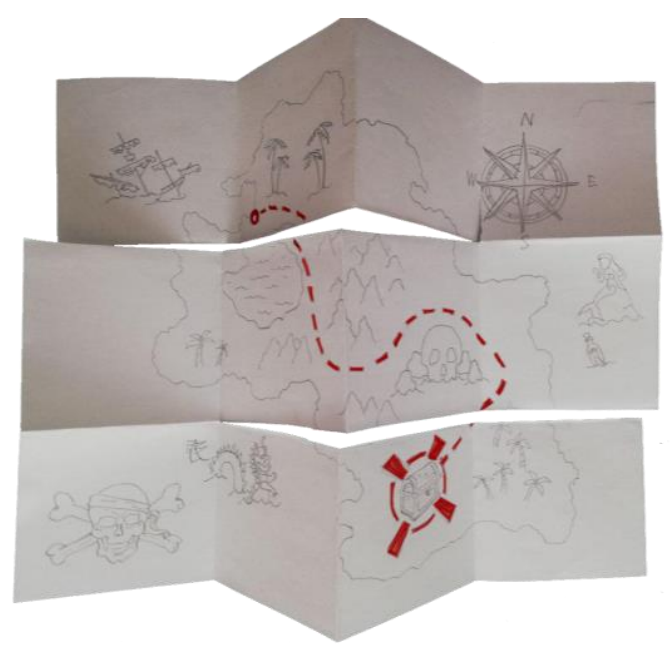

Ilustración 3: Ejemplo de libro del mapa del tesoro/ Imagen: Natalia Tello Burgos.

En este caso se parte de un formato algo más complejo en el que tras el diseño del mensaje o dibujo, se realizan determinados dobleces y cortes para obtener la composición final en la que el contenido quedará oculto tras su encuadernación. En este caso se les enseñan algunos ejemplos como el que se observa en la Ilustración 3 para que observen las posibilidades que proporciona esta tipología de libro y pongan en marcha su creatividad en función del tema que ellos escojan.

\subsection{El cuerpo del dragón}

Esta última actividad es la más avanzada y está dirigida al alumnado de entre 12 y 14 años. En este caso no se trata de hacer un trabajo individual, como en las anteriores, sino que cada uno de los formatos o módulos que realicen los estudiantes, se unirán al final del taller para formar un gran libro entre toda la clase.

Esta tipología de libro se asemeja bastante a los trabajos de origami o papiroflexia, aunque en este caso se utilizan a parte de las cartulinas de colores y materiales para el coloreado, también tijeras y pegamento. 


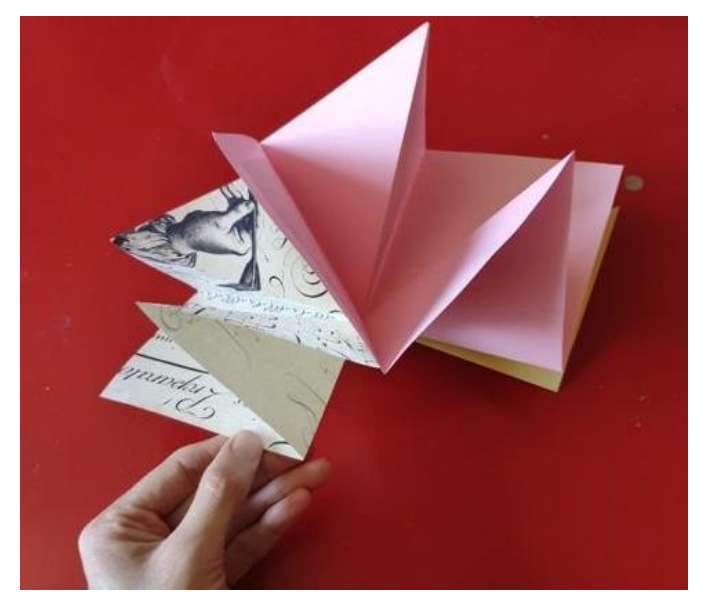

Ilustración 4: Ejemplo de libro del cuerpo del dragón/Imagen: Ana María López. Montes.

A partir de las figuras geométricas obtenidas por el alumnado participante surge el cuerpo de un dragón que, según se combinen las distintas formas y colores se obtendrán resultados muy variados de gran belleza y donde incluir diferente tipo de contenido según las características de cada taller impartido o la preferencia del alumnado que asiste (Ilustración 4).

Así, además de desarrollar su creatividad, el empleo de técnicas de origami sirve, como se ha demostrado en estudios precedentes para la adquisición de competencias en el ámbito de la geometría y de la adquisición de visión espacial (Barasona \& Gutiérrez, 2015), especialmente en educación secundaria donde ha demostrado ser un recurso muy positivo (Mayo, 2018).

\section{Talleres virtuales.}

Debido a la situación de emergencia sanitaria derivada del estado de alarma por COVID19, este año se nos ha planteado la necesidad de realizar otro tipo de actividades que puedan realizarse de manera no presencial. Coincidiendo con el día del libro, se ha elaborado una actividad práctica virtual para que los niños y niñas pudieran realizarla desde casa, supervisados por un adulto y con materiales caseros. En el archivo generado en .pdf los niños y adultos tienen a su disposición el listado de todos los materiales necesarios para efectuar la actividad, así como los diferentes pasos a seguir de manera clara y sencilla. Esta documentación se acompaña de ejemplos de mayor y menor dificultad que sirvan de modelo o inspiración para el trabajo a desarrollar de manera autónoma. 
Todas estas actividades se enfocaron fundamentalmente a niños y niñas de entre 3 y 6 años de edad, aunque en todos los casos pueden ser también realizadas por otras edades adaptando su dificultad.

La actividad está presentada en formato .pdf, en cuyo diseño se incluyen instrucciones precisas sobre los materiales necesarios, el procedimiento a seguir para conseguir cada tipo de libro e imágenes del resultado final que debería obtenerse. Este archivo se puede consultar y está disponible en la web ${ }^{1}$ del Vicerrectorado de Extensión Universitaria de la Universidad de Granada, dentro del taller días de libros, donde además de nuestras actividades propuestas Crea tu primer libro.3-4 años y Crea tu primer libro. 4-5 años, se pueden realizar otras propuestas por otros profesionales como Videocuentos o Recomendaciones de libros de literatura infantil y juvenil.

Además, para dar más difusión a este taller se han empleado diversas redes sociales entre las que destacar las oficiales de la Facultad de Bellas Artes de la Universidad de Granada, pero también Instagram, Facebook o Twitter. De esta manera el taller ha podido llegar a un gran público viendo como, incluso ayuntamientos o colegios de diferentes puntos de España, compartían las actividades con motivo del día del libro. Para disfrutar de las creaciones de todos los participantes en este taller, se ha creado un hashtag con el que todos han podido compartir sus trabajos.

\subsection{El bosque de...}

Siguiendo el mismo orden en la presentación de las actividades, esta primera está diseñada para los más pequeños (entre 3 y 4 años). Muy relacionada con la actividad del libro acordeón, los participantes, al tener la oportunidad de estar en casa y hacerlo con la ayuda de algún adulto, disponen de más tiempo para realizarla, así como de una mayor diversidad de materiales a utilizar. Este condicionante hace que en esta actividad se les invite especialmente a fomentar la creatividad, mezclando texturas, colores pegatinas, dibujos; en definitiva, diferentes técnicas o materiales con los que experimentar y aportar originalidad a sus creaciones.

\footnotetext{
${ }^{1}$ Enlace disponible en la siguiente URL https://educa.ugr.es/dias-de-libros/
} 
ISSN: 2340-9096

https://dx.doi.org/10.17561/rtc.extra2.5754

Una vez terminado, se invita a que cada persona que lo realice, personalice su propio cuento y su propio bosque (Ilustración 5).

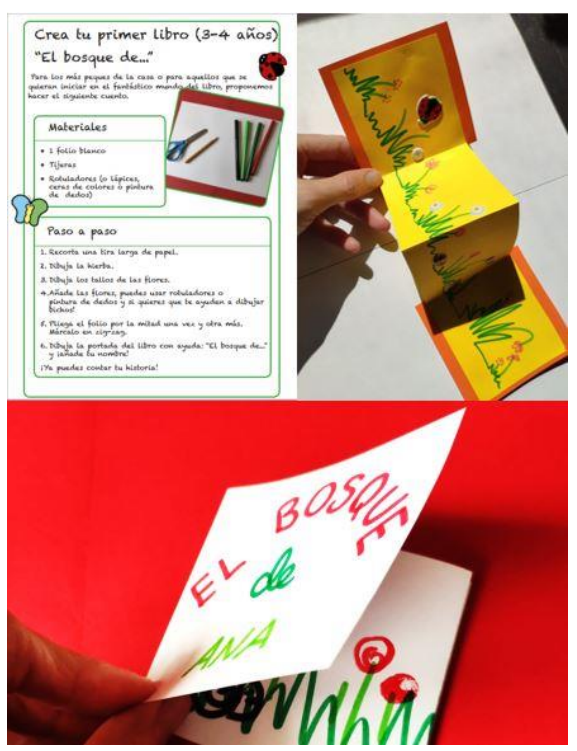

Ilustración 5: Secuenciación de los pasos para la construcción del libro bosque de... y libro acabado. Fuente: Actividades para el día del libro 2020.pdf

\subsection{Libro con animales}

La segunda actividad, dirigida a una franja de edad superior ( 5 a 6 años) se presenta como la creación de un libro con animales. Esta tarea propuesta resulta muy interesante pues se pueden añadir niveles de complejidad fácilmente para adaptarla a diferentes edades. En este caso se pueden trabajar de manera sencilla con diferentes formatos de base como: cartulinas, folios de colores, papeles coloreados... para crear diferentes capas o profundidades; así como añadir complejidad a los diseños creados (Ilustración 6).

Dado que dibujar un animal puede resultar complicado, en este ejercicio se invita a los participantes a dibujarlos con sus propios juguetes empleando la sombra que proyectan cuando se enfocan directamente con la luz de una lámpara o bien con la luz de sol. 
Revista de Estudios en Sociedad, Artes y Gestión Cultural

ISSN: $2340-9096$

https://dx.doi.org/10.17561/rtc.extra2.5754

Octubre 2020

Investigación

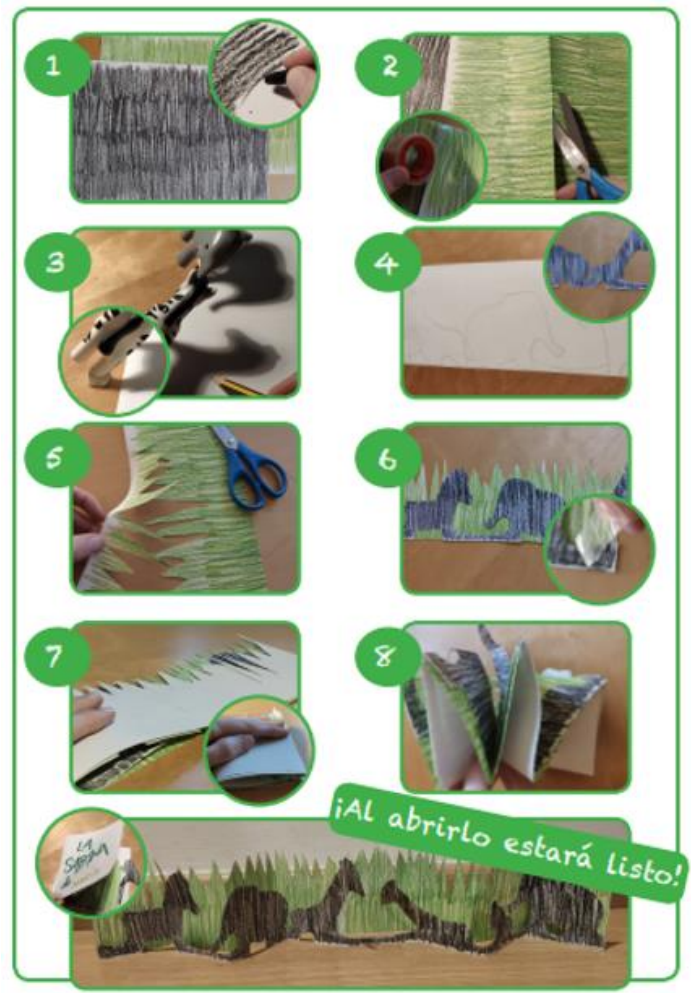

Ilustración 6: Secuenciación de los pasos para la construcción del libro con animales/ Fuente: Actividades para el día del libro 2020.pdf

Para que la actividad fuera más atrayente, se realizaron varios ejemplos de mayor y menor complejidad que sirvieran de modelo a aquellos que se animasen a llevar a cabo esta actividad en casa. De entre los ejemplos realizados, señalamos un libro con animales de la sabana que pensamos que podría ser interesante y atrayente debido a su parecido con el cuento y película Disney de El rey León, (Ilustración 7) empleando cartulinas de colores y efectuando planos diferenciados con siluetas más complejas. Por otra parte, dada las posibilidades que nos ofrecía esta actividad virtual, también se diseñó un $\underline{\text { ídeo }}^{2}$ corto en formato stopmotion colgado en YouTube con enlace directo desde la actividad generada y realizado con las sombras proyectadas de pitufos de juguete (Lucero, 2016).

\footnotetext{
${ }^{2}$ Enlace disponible en la URL https://www.youtube.com/watch?v=rlUHD8ZnOMO\&feature=youtu.be
} 
ISSN: $2340-9096$

https://dx.doi.org/10.17561/rtc.extra2.5754

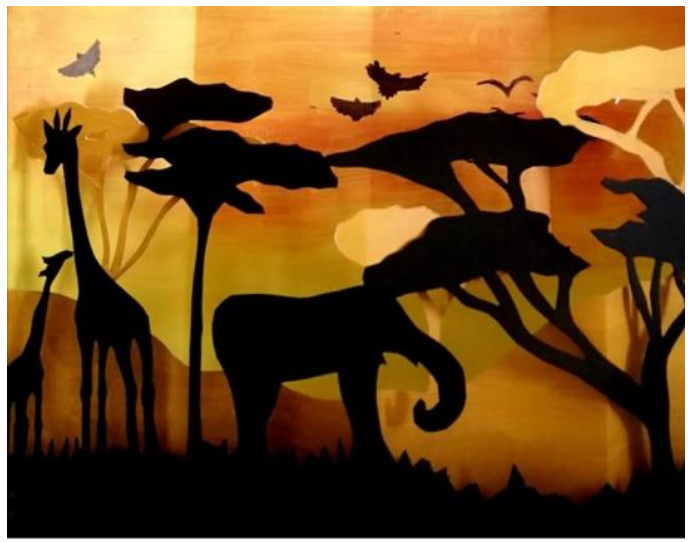

Ilustración 7. Ejemplo de libro con animales: La sabana/ Imagen: Ana Isabel Calero Castillo.

\section{Conclusiones}

A continuación, se exponen algunas de las reflexiones que hemos extraído de esta experiencia con los talleres a lo largo de estos últimos años.

Se han obtenido resultados muy positivos de este trabajo, gracias al interés y motivación de los niños y niñas a la hora de realizar las actividades, así como a la implicación del alumnado y egresados del Grado en Bellas Artes y en Restauración y Conservación y de Bienes Culturales de la Universidad de Granada.

El trabajo en conjunto, bien para crear obras personales pero dirigidas o piezas complementarias que formarían parte de un todo, han aportado seguridad y creencia de aceptación en el grupo a aquel alumnado señalado como tímido e introvertido. Así han sido capaces de contar la historia que guarda su creación frente a sus propios compañeros ante el asombro de su profesorado habitual, desarrollando su creatividad en todas las actividades, compartiendo materiales y fomentando el trabajo de tipo colaborativo.

Asimismo, consideramos que la implementación y desarrollo de estos talleres aporta grandes beneficios para los estudiantes entre los que se pueden destacar: la mejora de destrezas manuales a todos los niveles, la sensibilización y acceso al conocimiento acerca del patrimonio documental y cultural y finalmente, a la importancia de la conservación y defensa de nuestros libros. 


\section{Agradecimientos}

Agradecemos al Vicerrectorado de Extensión Universitaria de la Universidad de Granada por dar difusión a nuestro trabajo y alojar nuestras actividades en su web.

A todos los centros educativos (institutos y colegios) que han participado en nuestros talleres, así como a todo el alumnado por su colaboración en esta iniciativa.

Finalmente, a todas las compañeras de nuestro grupo de trabajo Teresa López, Ana Carrasco y Marta Durbán.

\section{Referencias}

Argüelles, J. D. (2018). Literacidad: Más allá de la decodificación textual. México: Campus Milenio Suplemento Universitario. Recuperado de http://campusmilenio.mx/index.php?option=com_k2\&view=item\&id=9655:literacidadmas-alla-de-la-decodificacion-textual \&Itemid=143

Barasona Villarejo, M. L.; Gutiérrez Rubio, D. (2015) Experiencia docente del uso del Origami para la mejora del análisis y visión espacial. Aula de Encuentro, 17, no 2. Recuperado de https://revistaselectronicas.ujaen.es/index.php/ADE/article/view/2661

Gil Flores, J. (2011). Hábitos lectores y competencias básicas en el alumnado de educación secundaria obligatoria. Educación XXI, pp. 117-134. ISSN: 1139-613X. Recuperado de http://e-spacio.uned.es/fez/eserv/bibliuned:EducacionXXI-2011-14-15060/Documento.pdf

López, J. M. C. (2014). El papel del patrimonio en los centros educativos: hacia la socialización patrimonial. Tejuelo: Didáctica de la lengua y la Literatura. Educación, (19), 76-96.

Lucero Martínez, J. A. (2016). Del libro de texto a YouTube; una aproximación a las nuevas tecnologías y a las nuevas formas de aprendizaje. RESED. Revista de Estudios Socioeducativos, (4) S2 pp. 185-187. Recueprado de http://hdl.handle.net/10498/18951 
Revista de Estudios en Sociedad, Artes y Gestión Cultural

ISSN: 2340-9096

https://dx.doi.org/10.17561/rtc.extra2.5754

Octubre 2020

Investigación

Mayo Rivera, R. (2018). El Origami aplicado a la educación. Unidad didáctica del bloque de expresión y comunicación técnica de $1^{\circ}$ de ESO. Trabajo Fin de Máster. Universidad de Valladolid.

Millán, J.A. (2017). La lectura en España. Informe 2017. Madrid: Federación de Gremios de Editores de España. ISBN: 978-84-86141-61-5. Recuperado de http://www.fge.es/lalectura/2017/default.html

Tacón Clavaín, J. (2009). La restauración en libros y documentos. Técnicas de intervención 